\title{
Eberbard Kolb
}

\section{Stabilisierung ohne Konsolidierung? Zur Konfiguration des europäischen Mächtesystems 1871-1914}

Der Friedensschluß am Ende des deutsch-französischen Krieges (Präliminarfrieden von Versailles 26. 2. 1871 und Frankfurter Friedensvertrag 10. 5. 1871) bedeutete in der Tat den Abschluß einer fast zwanzigjährigen Periode begrenzter Großmachtkriege in Europa. Zwischen 1854 und 1871 war jede der europäischen Großmächte in mindestens einen Krieg verwickelt, und in jedem dieser Kriege (mit Ausnahme des dänischen Krieges) standen Mächte der Pentarchie gegeneinander: im Krimkrieg Frankreich und Großbritannien gegen Rußland, im Krieg von 1859 Frankreich (und Piemont-Sardinien) gegen die Habsburgermonarchie, 1866 Preußen (mit Italien) gegen Österreich, 1870/71 Preußen-Deutschland gegen Frankreich. In diesem Zeitraum erfolgte eine einschneidende Veränderung des Staatensystems und der Machtlage in Kontinentaleuropa. Mit dem Deutschen Reich und dem Königreich Italien waren zwei - in dieser territorialen Gestalt „neue " - Staaten entstanden; Frankreich und die Habsburgermonarchie hatten gegenüber der Zeit vor 1870 deutlich an Machtgewicht eingebüßt, Rußland zumindest nicht einen erheblichen Machtzuwachs gewonnen. Ob das Deutsche Reich wirklich eine „halbhegemoniale“ Stellung errungen hatte und dadurch die „balance of power" in Europa nachhaltig erschüttert war, ist umstritten; gegenüber einer derartigen Interpretation ist eher Skepsis angebracht. Viel bemerkenswerter ist die Tatsache, daß entgegen den Erwartungen und Befürchtungen vieler Zeitgenossen das aus den kriegerischen Konflikten der 1850er und 1860 er Jahre hervorgegangene System sich als erstaunlich stabil erwies - über vierzig Jahre lang gab es keinen Krieg, in dem eine europäische Großmacht gegen eine andere stand (das Osmanische Reich, das nicht zur Pentarchie gehörte, bleibt dabei außer Betracht). Aber - so Peter Krüger - es war eine Stabilisierung ohne Konsolidierung, insofern habe es sich um eine nur scheinbare und deshalb trügerische Stabilität gehandelt; weil in den Jahrzehnten nach 1871 der „Neuaufbau einer kooperativen und sicheren europäischen Ordnung in der Form eines europäischen Konzerts, ähnlich dem, das auf dem Wiener Kongreß geschaffen worden war", unterblieb, ließen sich die aufgehäuften Konfliktpotentiale nicht bändigen, und es entwickelte sich sukzessive jene Mächtekonstellation, die den Ausbruch eines großen Krieges in 
Europa wenigstens auf längere Sicht nahezu unausweichlich machte. So kam es schließlich zum Ersten Weltkrieg, der „Ur-Katastrophe“ des 20. Jahrhunderts (George F. Kennan).

Ist das europäische System der Jahre 1871 bis 1914 mit der Formel „Stabilisierung ohne Konsolidierung " treffend charakterisiert? Und worin hätte - unter den Bedingungen dieser Jahrzehnte - eine „Konsolidierung“ bestehen können oder müssen? Peter Krüger umreißt eindringlich vier Ursachenbündel, vier „Ausgangspunkte" für die wachsende Unruhe und strukturelle Unsicherheit des Staatensystems: erstens die folgenreichen Veränderungen der politischen Landkarte Europas ohne europäische Übereinkunft und Sanktionierung; zweitens die in allen Staaten zunehmende Tendenz, mit allen zu Gebote stehenden Mitteln, insbesondere auch durch eine forcierte Aufrüstung, die eigene Machtposition zu stärken, weil eine europäische Absicherung nicht mehr funktionierte; drittens die "wachsende Bedeutung der inneren Organisation der Staaten, ihrer Verfassung, ihres politischen Systems und ihrer Gesellschaftsordnung", so daß die Verfassungsentwicklung in den einzelnen Staaten immer größere Bedeutung für das außenpolitische Agieren gewann; viertens schließlich die Tatsache, daß aus dem Friedensschluß (von 1871) als solchem Kriegsgefahr erwuchs, insbesondere auch aufgrund fehlender europäischer Sanktionierung und Garantierung des neuen $\mathrm{Zu}-$ stands.

Gewiß sind damit wesentliche Charakteristika des Mächtesystems und der Mächtepolitik im Europa des späten 19. Jahrhunderts benannt. Doch diese Faktoren kennzeichnen nicht das "Neue" der Situation ab 1871, sie waren alle schon vor 1871 wirksam. Mit Recht spricht Peter Krüger von „folgenreichen Veränderungen des Staatensystems seit der Mitte des 19. Jahrhunderts", die der auf dem Wiener Kongreß geschaffenen curopäischen Ordnung den Todesstoß versetzten. Dies soll durch einige skizzenhafte Hinweise verdeutlicht werden.

Eine so tiefgreifende und folgenreiche Modifizierung der politischen Landkarte Europas wie die Verdrängung der Habsburgermonarchie aus Italien (1859/1866) und die Entstehung des Königreichs Italien erfolgte ebenso wenig im Rahmen eines europäischen Kongresses wie die Annexion Savoyens und Nizzas durch Frankreich (1860). Man kann bestenfalls von einer stillschweigenden Sanktionicrung dieser territorialen Veränderungen durch die übrigen Großmächte sprechen, wie dann ja auch das Ergebnis des deutsch-französischen Krieges, vor allem die Annexion des Elsaß und Deutsch-Lothringens, von den Mächten stillschweigend sanktioniert worden ist. Daß alle Großmächte verstärkt den Ausbau der eigenen Machtressourcen betrieben, sich am Postulat der „Realpolitik“ orientierten und völlige außenpolitische Handlungsfreiheit zu gewinnen suchten, sind Spezifika bereits der Zeit vor 1870. Der von Krüger konstatierte "fundamentale Auflockerungs- und Wandlungsprozeß der europäischen Staatengemeinschaft" vollzog sich in der Tat seit Beginn des Krimkriegs.

Auch das Revancheverlangen als Triebkraft bei der außenpolitischen Positionierung eines Staates ist keineswegs ein Phänomen, das in einzigartiger Weise die internationale Politik nach 1871 bestimmt. Nach jedem Friedensschluß ist es das 
Bestreben des Unterlegenen oder Unzufriedenen, eine Revision des Friedens zu erreichen, sei es als Nahziel, sei es als Fernziel der eigenen Außenpolitik. Verständlicherweise sind Äußerungsintensität und Äußerungsformen des Revanchewillens abhängig von der Gesamtheit der Umstände, differieren daher von Fall zu Fall.

Nach dem Pariser Frieden von 1856, der Rußland die Pontusklauseln und die Abtretung Bessarabiens auferlegte, betrieb das Zarenreich eine „revisionistische" Außenpolitik. Wenn die Revisionsabsicht auch nicht ein Anliegen breitester Volksschichten gewesen sein mag, so bestimmte sie doch zweifelsfrei die Haltung der russischen Führungsschicht. Das russische Kabinett beurteilte die jeweiligen Konstellationen in den internationalen Bezichungen unter dem Gesichtspunkt, inwieweit sie eine Revision des Pariser Friedens ermöglichen oder erleichtern konnten. Die Revision erfolgte dann in zwei Etappen: 1870/71 wurde die Aufhebung der Pontusklauseln erzwungen, 1878 gewann Rußland Bessarabien zurück. Auch der Habsburgermonarchie waren nach 1859 und erst recht nach 1866 revisionistische Tendenzen nicht fremd: Nach $1859 / 60$ bestand zumindest in Kreisen der österreichischen Führungsschicht eine Neigung, die Lombardei wiederzugewinnen, falls die gesamteuropäische Situation dies erlauben sollte (vgl. die Schönbrunner Gespräche zwischen Rechberg und Bismarck im August 1864), und dic Entschlossenheit der österreichischen Regierung, die „Lösung von 1866“ bei nächstbester Gelegenheit zu revidieren, steht außer Frage und bildete einen wichtigen Faktor in der Mächtepolitik der Jahre 1866 bis 1870 . Selbst Dänemark hat die Bestimmungen des Friedensschlusses von 1864 nicht als letztes Wort in der Sache betrachtet; jede europäische Spannungssituation wurde vom Kopenhagener Kabinett auf Revisionschancen abgetastet (vgl. 1870).

Mit diesen Bemerkungen soll lediglich akzentuiert werden, daß das französische Revancheverlangen nach 1870 nicht ein strukturell neues Element in den internationalen Beziehungen darstellt. Nur was Dimension, Verankerung in breiten Bevölkerungsschichten und Wirkungsmächtigkeit angeht, hob sich das französische Revanchebedürfnis deutlich von den revisionistischen Bestrebungen vor 1870 in anderen Ländern $\mathrm{ab}$.

Wenn sich somit ergibt, daß die von Peter Krüger bezcichneten Wesensmerkmale der prekären Lage in Europa ab 1871 bereits in den internationalen Beziehungen der Jahre seit Mitte des 19. Jahrhunderts anzutreffen sind, also nicht kausal und nicht ausschließlich auf den deutsch-französischen Krieg und den Frankfurter Frieden zurückgeführt werden können - worin bestand dann das „Neue“ in der Konfiguration des europäischen Mächtesystems ab 1871? Hier sind, wie ich meine, vor allem zwei Elemente zu nennen.

Daß es über vier Jahrzehnte lang in Europa nicht zum „großen Krieg“ kam, ist nicht das Ergebnis schieren Zufalls oder ausschließliches Verdienst der Staatsmänner. Mochte nach 1871 der europäische Frieden auch mehrfach akut bedroht sein (und noch öfter bedroht erscheinen), so war der Frieden - so paradox das klingen mag - gegenüber der Zeit vor 1870 tatsächlich strukturell sicherer geworden, ungeachtet des von Zeit zu Zeit sich erhebenden Kriegsgeschreis in Presse und öf- 
fentlicher Meinung, ungeachtet auch der häufigen Apostrophierung möglicher Kriegsgefahr in der diplomatischen Korrespondenz aller europäischen Kabinette. Strukturell sicherer aus zwei Gründen.

Erstens: Zwei der großen Unruhepotentiale der vorausgehenden Jahrzehnte existierten nicht mehr - die "deutsche Frage" war ebenso "gelöst" wie die italienische. Der auf den Erwerb von Trentino und Trieste/Küstenland gerichtete italienische Irredentismus erwies sich als nicht so stark und akut, daß er in den internationalen Beziehungen konstellationsbildend wirksam werden konnte. Einen auf die deutschen Provinzen Österreichs zielenden deutschen Irredentismus gab es nicht. Insoweit war die Mitte Europas konsolidiert, zumal seit dem Krieg von 1870 von der polnischen Frage - die ebenfalls über Jahrzehnte hin ein Potential der Unruhe sowie der potentiellen und akuten Störung der Mächtebeziehungen gebildet hatte - keine Sprengkraft mehr auf das Mächtesystem ausging.

Zweitens: Spätestens das Geschehen von 1870/71 hatte allen europäischen Staatsmännern deutlich zum Bewußtsein gebracht, daß die Zeit vorbei war, in der eine der europäischen Großmächte es riskieren konnte, gegen eine andere Krieg in Form eines „Zweikampfs“ zu führen, wie das noch 1859, 1866 und 1870 geschehen war. 1870 hatte die französische Staatsführung den Krieg gegen Preußen als Zweikampf begonnen - wenn auch in der Hoffnung und Erwartung einer Unterstützung durch Österreich-Ungarn und Italien, so doch ohne vertragsmäßige $\mathrm{Zu}-$ sicherung eines derartigen Beistands. Nach 1871 war der Gedanke, Frankreich könne allein auf sich gestellt das Deutsche Reich bekriegen, völlig illusorisch; das wußten die französischen Politiker ganz genau, und auch publizistische Tiraden vermochten diesen Sachverhalt nicht zu vernebeln. Eine ähnliche Feststellung läßt sich für die anderen Großmächte treffen: Keine Macht der Pentarchie konnte ernsthaft das außenpolitische Ziel verfolgen, eine andere Großmacht in einem Zweikampf niederzuwerfen (etwa Rußland die Habsburgermonarchie oder umgekehrt).

Das bedeutete aber, daß nun die Bündnispolitik einen viel höheren Stellenwert, ja eine neue Qualität gewann: ohne kriegsfähige Koalition kein europäischer Krieg. Eine kriegsfähige Koalition war jedoch kaum als ad hoc-Koalition zu bilden; es bedurfte dazu langfristiger bindender Abmachungen und der Herstellung formeller Bündnisse. Es konnte zwar viel Zeit vergehen, bis das eine oder andere Bündnis konkrete Gestalt annahm, aber wenn dann tatsächlich der Ernstfall eintrat, mußte die durch Bündnisverpflichtungen eingeschränkte Handlungsfreiheit der politischen Akteure verheerende Auswirkungen zeitigen: Krieg war nun gleichbedeutend mit dem großen europäischen Krieg.

Welche Prämissen lagen Bismarcks Bündnispolitik der 1870er und auch noch der 1880er Jahre zugrunde? Überzeugt, das junge Deutsche Reich sei saturiert und müsse im Interesse der eigenen außenpolitischen Staatsräson eine friedenswahrende Politik betreiben, richtete Bismarck seine Außenpolitik ganz darauf aus, prophylaktisch die einzige für das Deutsche Reich existentiell bedrohliche Konstellation zu verhindern, nämlich eine Konflagration zwischen Rußland und Österreich wegen der Balkanfragen; eine Konstellation, bei der Deutschland zu 
optieren gezwungen sein würde und bei der auch Frankreich ins Spiel kommen konnte; nur unter dieser Voraussetzung vermochte das französische Revanchebedürfnis dem Deutschen Reich gefährlich zu werden. Möglichste Entschärfung des russisch-österreichischen Konfliktpotentials, insbesondere in der Risikozone Balkan - das war ein gelegentlich ausgesprochener, immer aber unausgesprochen handlungsleitender Gesichtspunkt in Bismarcks Außenpolitik. Der Grundgedanke von Bismarcks "System“ war insofern von verblüffender - und grandioser - Einfachheit; ihn im raschen Wandel der europäischen Großwetterlage in praktische Außenpolitik umzusetzen, dazu bedurfte es allerdings subtiler diplomatischer Techniken und einer ständigen angestrengten Beobachtung, in welcher Weise sich die Beziehungen zwischen den Mächten jeweils akzentuierten. Eine flexible, defensive Bündnispolitik betrieb Bismarck seit dem Ende des deutschfranzösischen Krieges, nicht erst ab 1875. Die Meinung, das Jahr 1875 („Krieg-inSicht“-Krise) markiere die eigentliche „Wegscheide“ der europäischen Mächtepolitik, findet in den Quellen keine Stütze ${ }^{1}$.

In den beiden Jahrzehnten vor 1914 erfuhr der Charakter der Bündnispolitik zwischen den Großmächten und damit die Konfiguration des europäischen Staatensystems eine grundlegende Veränderung. An die Stelle der bis dahin noch einigermaßen flexiblen Beziehungen zwischen den europäischen Mächten trat nun so läßt es sich thesenhaft formulieren - die Formierung von Mächteblöcken, die sich mit zunehmender Feindseligkeit gegenüberstanden; kurze Phasen der Deeskalation vermochten die konfrontative Blockbildung, hier die Mittelmächte, dort die Staaten der Entente, nicht mehr aufzuheben. Dominanz stark offensiver Komponenten, Ausdehnung der Mächterivalitäten auf außereuropäische Konfliktfelder, Bündnisautomatik und Fatalismus der leitenden Staatsmänner wurden zu beherrschenden Merkmalen der europäischen Mächtepolitik am Vorabend des Ersten Weltkriegs.

Ist der Ausbruch des Weltkriegs auf eine unterbliebene „Konsolidierung“ des europäischen Staatensystems zurückzuführen, die möglich gewesen wäre, die aber

1 Ich spiele an auf Andreas Hillgrubers wirkungsmächtige Interpretation der Krise von 1875: Die „Krieg-in-Sicht“-Krise - Wegscheide der Politik der europäischen Großmächte in der späten Bismarckzeit, in: Ernst Schulin (Hrsg.), Gedenkschrift Martin Göhring (Wiesbaden 1968) 239-253. Der aus einem Vortrag erwachsene Festschriftbeitrag (Kernformulierungen dann auch, teilweise wörtlich, in: ders., Bismarcks Außenpolitik (Freiburg 1972) 129ff., (Neuauflage 1993) 115ff.) beruht auf einer Auswertung der damals (1968) „neueren Literatur"; archivalische Quellen wurden nicht herangezogen, die gedruckten Quellen nur sehr selektiv. Mit der ihm eigenen Kraft der Zuspitzung entwickelte Hillgruber die Auffassung, Bismarck habe nicht gleich nach der Reichsgründung die „bisher ja recht große Variationsbreite seiner außenpolitischen Methoden radikal eingeschränkt und nunmehr eingleisig im Sinne einer den einmal erreichten Besitzstand der europäischen Staatenwelt unbedingt wahrenden Politik taktiert"; erst durch die Krise von 1875 sei er zur Einsicht gelangt, daß eine Veränderung der bestehenden Kräfteverhältnisse in Mitteleuropa ohne großen Krieg nicht mehr möglich sei. Hillgrubers suggestive Deutung, die nicht aus der Analyse der Quellen abgeleitet ist, sondern cine Konstruktion darstellt, beeinflußt noch jüngste Forschungen, weil die Quellen durch die Brille der Hillgruberschen Interpretation gelesen werden. Aus diesem Grunde scheint es an der Zeit, Hillgrubers Beurteilung der Krise von $1875 \mathrm{zu}$ überprüfen. 
leichtfertigerweise nicht in Angriff genommen wurde? Peter Krüger betrachtet als den angemessenen Weg zur Konsolidierung des Staatensystems nach 1871 bzw. als die dazu geeigneten Instrumente die „Wiederaufrichtung eines auf gemeinsame vertragsmäßige Bindungen und Verpflichtungen festgelegten Verfahrensweges und europäischen Konzerts". So wünschbar dies gewiß gewesen wäre, so bestehen doch erhebliche Zweifel, ob ein derartiges retrospektives Rezept als realistisch unter den Bedingungen der Zeit bewertet werden kann, ob die Wiederherstellung eines europäischen Konzerts von den damaligen Lenkern der Außenpolitik aller Großmächte, von den maßgebenden politischen Akteuren als ebenso vordringliche wie konkrete Aufgabe erkannt wurde. Bei den oben bezeichneten Ursachenkomplexen für die um die Mitte des 19. Jahrhunderts einsetzende "wachsende Unruhe und Unsicherheit des Staatensystems" hat es sich wohl doch um säkulare Trends von außerordentlicher Mächtigkeit gehandelt; sie aufzuhalten oder gar umzusteuern, dürfte außerhalb der Möglichkeiten einzelner Staatsmänner gelegen haben, die ja unter den gegebenen Verhältnissen zu operieren hatten und ihr Agieren nicht auf die ideale Konzeption eines wiederaufzurichtenden europäischen Konzerts im Geist der Wiener Ordnung ausrichten konnten. Unter diesen Umständen blieb nur das Bemühen um Abbau der Konfliktpotentiale und um Risikobegrenzung eine realistische Zielsetzung. Es läßt sich darüber hinaus die Frage aufwerfen, ob das internationale System nicht überfordert wird, wenn man seine Qualität mit der Elle dauerhafter Konsolidierung mißt. Ein langfristig konsolidiertes Mächtesystem ist eher die seltene Ausnahme, nicht die Regel. Unter den Gegebenheiten der Vorweltkriegszeit und angesichts der damals vorherrschenden Einstellungen sehe ich kaum Chancen für eine Akzeptanz des Programms, zu einer neuen Übereinkunft der Großmächte zu gelangen, „alle wesentlichen europäischen Streitfragen gemeinsam zu behandeln, und zwar auf der Basis gemeinsam sanktionierter Verträge, in Anerkennung bestimmter Verhaltensnormen, in denen die Grenzen des Erlaubten in der Ausnutzung des Gesamtsystems zu eigenen Zwecken zum Ausdruck kamen, und in der Ausgestaltung gewisser Instrumentarien der Konsultation und Konfliktregelung, wie immer sie im einzelnen aussehen mochten“.

Welche gangbaren Wege zum Abbau des brisanten Konfliktpotentials hätte es dann vor 1914 gegeben? Wenn es zutrifft, daß das wesentliche Kennzeichen der Konfiguration im europäischen Mächtesystem ab 1890 die Herausbildung und zunehmende Verfestigung von Blöcken war und von dieser Blockbildung fortdauernde Gefahren für die Erhaltung des europäischen Friedens ausgingen, dann mußte eine auf Friedenswahrung zielende Politik sich bemühen, die Tendenz zur Zementierung von Mächteblöcken aufzulockern und so Manövrierfähigkeit für das außenpolitische Agieren zurückzugewinnen. Das war gewiß schwierig genug, aber unter den Bedingungen der Zeit eine wohl eher realisierbare Zielperspektive als die Wiederaufrichtung eines europäischen Konzerts, wie es in den Jahrzehnten nach 1815 bestanden hatte. Und wenn in der Zeit vor 1914 die Politik der Kabinette auch flankiert wurde durch nationalistische Emotionen und ausgeprägte Feindbilder in einer breiten Öffentlichkeit aller Staaten (und dadurch die Front- 
stellungen untermauert wurden), so darf man andererseits doch den Blick nicht ausschließlich auf das richten, was Mächte und Völker in Europa trennte. Neben der Tendenz zur Verfestigung der Blöcke gab es in der Zeit vor 1914 auch einen Prozeß zunehmender internationaler Verflechtung, sich intensivierender blockübergreifender Handelsbeziehungen und gemeinsamer internationaler Regelungen auf vielen Gebieten (Verkehr, Kommunikationswesen usw.). Der Vorweltkriegszeit haftet somit durchaus etwas Ambivalentes an; Bemühungen um die Erhaltung des europäischen Friedens, um Auflockerung statt Befestigung der Blöcke, um Abbau statt Potenzierung des Konfliktpotentials mußten deshalb nicht von vornherein zum Scheitern verurteilt sein.

Gerade in dieser Hinsicht ist der deutschen Außenpolitik vor 1914 völliges Versagen vorzuwerfen. Die Abkehr von den Einsichten, die der Bismarckschen Politik zugrundelagen (flexible Bündnispolitik mit dem Ziel der dem nationalen Interesse Deutschlands dienlichen Friedenswahrung), hat sich bitter gerächt. Statt mit allen Kräften der Formierung von Mächteblöcken entgegenzuwirken und die eigene außenpolitische Handlungsfreiheit zu behaupten, haben die Leiter der deutschen Außenpolitik - in leichtfertiger Überschätzung des deutschen Machtpotentials und in Verkennung der gesamteuropäischen Mächtekonfiguration - die Existenz sich feindlich gegenüberstehender Mächtekoalitionen fatalistisch hingenommen, auf dieser Grundlage ihren außenpolitischen Kurs konzipiert und schließlich die Unausweichlichkeit einer großen europäischen Konflagration zunehmend zum Fixpunkt ihres politischen Kalküls gemacht. 
